Artículo

\title{
Terra: sistema para proyectos de nivelación de tierras con topografía regular o variable
}

\author{
Francisco García Herrera ${ }^{1 \S}$ \\ Jesús Chávez Morales ${ }^{1}$ \\ Juan Enrique Rubiños Panta ${ }^{1}$ \\ María Liliana Terrazas Onofre ${ }^{2}$ \\ ${ }^{1}$ Colegio de Postgraduados-Campus Montecillo-Hidrociencias. Carretera México-Texcoco km 36.5, Estado \\ de México. CP. 56230. Tel. 595 9520200. (chavezje@colpos.mx; jerpkike@colpos.mx). ${ }^{2}$ Colegio \\ Mexicano de Especialistas en Recursos Naturales. De las flores \#8, San Luis Huexotla, Texcoco, Estado de \\ México. CP. 56220. Tel. 595 9285236. (terrazas.liliana88@gmail.com).
}

${ }^{\S}$ Autor para correspondencia: fragarhe@gmail.com.

\section{Resumen}

La nivelación de tierras es indispensable para reducir el volumen de agua aplicado y aumentar la uniformidad del riego por gravedad. Se presenta el sistema de cómputo electrónico Terra, para el cálculo de proyectos de nivelación de terrenos agrícolas, útil para examinar innumerables alternativas de diseño en un lapso muy corto de tiempo. Los datos de campo con que se alimenta, pueden ser puntos obtenidos con un levantamiento topográfico de una cuadrícula rectangular o de un levantamiento de distribución variable (radiaciones). El cálculo de volúmenes de corte y relleno se realiza usando 'el método de los cuatro puntos' apoyado con el método de Kriging en el caso de la topografía de distribución variable. El algoritmo desarrollado es capaz de dividir el terreno en tablas para su nivelación independiente, con el objetivo de reducir volúmenes de corte y relleno impactando directamente en el costo de nivelación. Se generan planos de la topografía del terreno antes y después de la nivelación; además de planos de cortes y rellenos y un diagrama de bloques como guía para el tractorista en el proceso de nivelación.

Palabras clave: DEM, isolineas, Kriging.

Recibido: junio de 2020

Aceptado: septiembre de 2020 


\section{Introducción}

De acuerdo con CONAGUA (2014) de la superficie de riego en México, 90\% tiene problemas de eficiencias de aplicación bajas, que pueden mejorarse con la nivelación de tierras si las pendientes topográficas son adecuadas, lo que implica una mayor uniformidad del riego y una reducción del volumen de agua aplicado (Saucedo et al., 2006; Fuentes y Rendon, 2012).

La nivelación de tierras se justifica en cualquier proyecto de irrigación, ya que generalmente se invierten sumas considerables en obras de captación y distribución, comparativamente, se hacen bajas inversiones en la parcela, que es donde se refleja la bondad de todo un complejo sistema de irrigación (Walker, 1987). Se han desarrollado muchos programas de computadora relativos al tema de la nivelación de terrenos agrícolas, buscando siempre ahorrar tiempo en el diseño y realizar los cálculos óptimos con el menor costo posible (Covarrubias, 1968).

Dentro de los primeros programas en México se tiene a Nivterra v1.0 desarrollado para computadoras personales en Fortran 77, con mejoras posteriores en Pascal para su versión 2.0 (Chávez et al., 1990), dando origen a sistemas que se usan hoy en día en la práctica, como el Sinivet en sus distintas versiones utilizado para levantamientos por cuadrícula y para la nivelación sin divisiones (Hernández y Sánchez, 2008). Los pocos programas existentes en la actualidad para el diseño de proyectos de nivelación de tierras, usa como fuente de datos levantamientos realizados por cuadrícula. Como resultado de varios años de trabajo en el área de desarrollo de software aplicado a la nivelación de tierras se desarrolló el programa Terra 1.0, para procesar datos de campo que se obtienen con levantamientos topográficos con el método tradicional de levantamiento con puntos trazados en una cuadrícula rectangular o con una distribución variable (radiaciones).

Terra, busca adaptarse a las nuevas tecnologías que implican la toma de información de los datos de campo, para el uso y aplicación de la nivelación de tierras. El objetivo fue, el desarrollo de un sistema de cómputo electrónico, que incluye métodos de levantamiento topográfico con equipos modernos, como estaciones totales o GPS de precisión, que auxilie en el cálculo preciso y expedito para la elaboración de proyectos ejecutivos de nivelación de tierras agrícolas. El manejar una masa de puntos tomados por radiaciones en lugar de una cuadrícula, implica generar un modelo digital de elevaciones (MDE) que describa con alta confiabilidad el predio caracterizado. Esto se logra en Terra usando el método de Kriging para el modelado del terreno.

\section{Materiales y métodos}

La metodología incluye la aplicación de la interpolación espacial usando el método de Kriging (Villatoro et al., 2008) para la determinación de los pesos o factores de ponderación en el cálculo, estimación y cuantificación de volúmenes de corte y relleno y trazo de curvas de nivel del terreno antes y después de la nivelación, que cubren áreas que el topógrafo no lo hace en una cuadrícula homogéneamente espaciada en el procedimiento tradicional.

La solución del método de Kriging permite definir por el usuario un variograma de elevaciones en función del mejor ajuste realizado a su semivariograma experimental (Clark, 1979). Los modelos utilizados para el ajuste de los variogramas son: lineal, cuadrático, esférico, exponencial y gaussiano, con o sin el efecto pepita (Delhome, 1978). Al igual que Walker (1987); Cano (1997), 
Terra 1.0 incluye la división del terreno en tablas independientes propuestas por Marr (1957), en caso de que las pendientes del predio sean heterogéneas. En la Figura 1, se describe en forma simplificada el algoritmo usado para el desarrollo del sistema Terra 1.0.

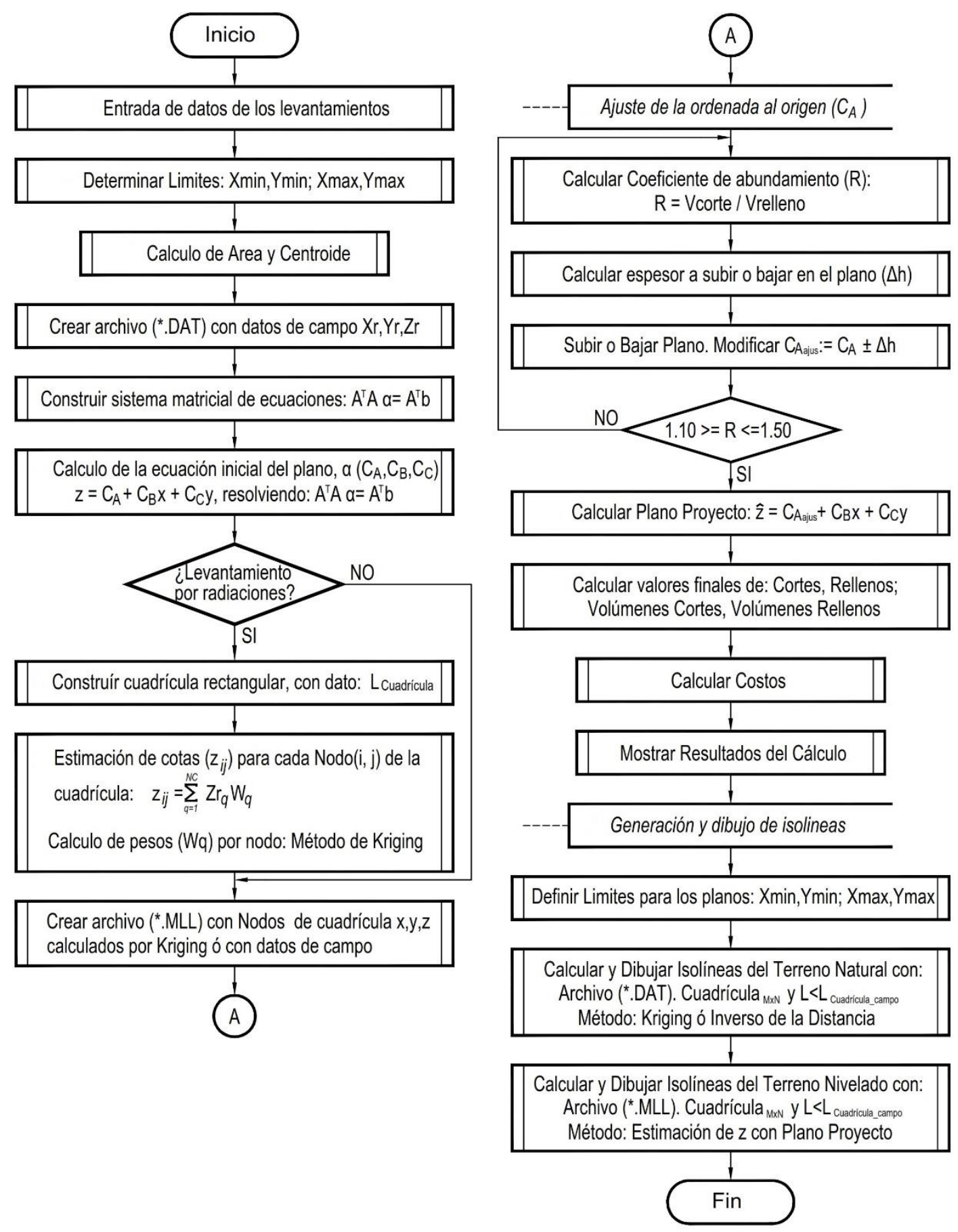

Figura 1. Algoritmo de nivelación, implementado en Terra para calcular el plano proyecto, sin divisiones o en una sola tabla. 


\section{Definición de los datos de los levantamientos}

Dependiendo del tipo de levantamiento topográfico realizado se obtuvieron coordenadas de campo para cada punto medido, ya sea en cuadrícula rectangular usando la propuesta de Marr (1957), en cuyo caso ya son coordenadas $\left(\mathrm{x}_{\mathrm{i}}, \mathrm{y}_{\mathrm{j}}, \mathrm{z}_{\mathrm{ij}}\right)$, donde $\mathrm{i}=1,2, \ldots, \mathrm{n} ; \mathrm{j}=1,2, \ldots, \mathrm{m} ; \mathrm{n}=$ número de hileras y $\mathrm{m}=$ número de columnas de la cuadrícula o radiaciones, en cuyo caso son coordenadas $\left(\mathrm{Xr}_{\mathrm{k}}, \mathrm{Yr}_{\mathrm{k}}\right.$, $\mathrm{Zr}_{\mathrm{k}}$ ). Donde: $\mathrm{k}=1,2, \ldots, \mathrm{P}$ y $\mathrm{P}=$ número de puntos levantados por radiaciones; las cuales se introducirán el cuadro de diálogo del programa, activando la opción de captura correspondiente según el levantamiento realizado. De la misma forma se introducen las coordenadas de cada uno de los vértices de la poligonal del terreno con sus respectivas coordenadas $\left(X_{v}, Y_{v}\right)$. Donde: $v=1$, $2, \ldots, \mathrm{NV}$ y $\mathrm{NV}=$ número de vértices de la poligonal del terreno.

\section{Límites de trabajo, área y centroide}

Con la información de los puntos de campo capturada, se determinan los límites extremos de una zona rectangular para el trabajo del proyecto de nivelación, definida por $\left(\mathrm{X}_{\min }, \mathrm{Y}_{\min }\right)$ en la esquina inferior izquierda y $\left(\mathrm{X}_{\text {máx }}, \mathrm{Y}_{\text {máx }}\right)$ en la esquina superior derecha. Para el caso de un levantamiento por radiaciones se crean los nodos de una cuadricula de dimensiones mxn en la zona rectangular definida, con una distancia entre nodos ( $\left.\mathrm{L}_{\text {cuadrícula }}\right)$, así para cada nodo se tiene información $\left(\mathrm{x}_{\mathrm{i}}, \mathrm{y}_{\mathrm{j}}\right)$, faltando estimar $\mathrm{z}_{\mathrm{ij}}$. Con las coordenadas de los vértices de la poligonal se calcula el área y el centroide del terreno $\left(\mathrm{x}_{\mathrm{c}}, \mathrm{y}_{\mathrm{c}}, \mathrm{z}_{\mathrm{c}}\right)$.

\section{Cálculo del plano proyecto}

Éste se realiza por mínimos cuadrados ordinarios mediante una solución matricial como la propuesta por Montgomery y Runger (2008), en la cual es indistinta la forma en que se realizó el levantamiento topográfico, la ecuación del plano proyecto inicial es: $\hat{\mathrm{z}}_{\mathrm{ij}}=\mathrm{C}_{\mathrm{A}}+\mathrm{C}_{\mathrm{B}} \mathrm{x}_{\mathrm{i}}+\mathrm{C}_{\mathrm{C}} \mathrm{y}_{\mathrm{j}}(1)$. Donde: $\hat{\mathrm{z}}_{\mathrm{ij}}=$ cota estimada del plano proyecto en un punto de coordenadas $\left(\mathrm{x}_{\mathrm{i}}, \mathrm{y}_{\mathrm{j}}\right)$, en $\mathrm{m} ; \mathrm{C}_{\mathrm{A}}=$ constante que geométricamente representa la cota del plano proyecto, en el origen del sistema de coordenadas, en $\mathrm{m} ; \mathrm{C}_{\mathrm{B}}=$ pendiente promedio del terreno en el sentido del eje $\mathrm{X}$, en dec; $\mathrm{C}_{\mathrm{C}}=$ pendiente promedio del terreno en el sentido del $\mathrm{Y}$, en dec; $\mathrm{x}_{\mathrm{i}}=$ coordenada $\mathrm{X}$ de la hilera $\mathrm{i}$, en $\mathrm{m}$; $\mathrm{y}_{\mathrm{j}}=$ coordenada $\mathrm{Y}$ de la columna $\mathrm{j}$, en $\mathrm{m} ; \mathrm{i}=1,2,3, \ldots, \mathrm{n} ; \mathrm{j}=1,2,3, \ldots, \mathrm{m} ; \mathrm{n}=$ número de hileras; $\mathrm{m}=$ número de columnas. Guajarati y Porter (2010), proponen calcular los coeficientes de un plano con: $\left.\left.\left(A^{T} A\right) \hat{\beta}=A^{T} b \quad 2\right) ; \quad\left(A^{T} A\right)=\left[\begin{array}{ccccc}1 & 1 & 1 & \ldots & 1 \\ X_{1} & X_{2} & X_{3} & \ldots & X_{n} \\ Y_{1} & Y_{2} & Y_{3} & \ldots & Y_{n}\end{array}\right]\left[\begin{array}{cccc}1 & X_{1} & Y_{1} \\ 1 & X_{2} & Y_{1} \\ 1 & X_{3} & Y_{1} \\ \vdots & \vdots & \vdots \\ 1 & X_{n} & Y_{n}\end{array}\right] \quad 3\right)$; $\left.\left(A^{T} b\right)=\left[\begin{array}{ccccc}1 & 1 & 1 & \cdots & 1 \\ X_{1} & X_{2} & X_{3} & \cdots & X_{n} \\ Y_{1} & Y_{2} & Y_{3} & \cdots & Y_{n}\end{array}\right]\left[\begin{array}{c}Z_{1} \\ Z_{2} \\ Z_{3} \\ \vdots \\ Z_{n}\end{array}\right] \quad 4\right)$

Donde: $\mathrm{A}=$ matriz de coordenadas $\mathrm{XY} ; \mathrm{A}^{\mathrm{T}}=$ transpuesta de la matriz de coordenadas $\mathrm{XY} ; \mathrm{b}=$ vector de coordenadas $Z ; \widehat{\beta}=$ vector de incógnitas o coeficientes $\left(C_{A}, C_{B}, C_{C}\right)$. De la ecuación (2) se despeja el vector de incógnitas o vector de coeficientes $(\widehat{\beta})$, para obtener los valores de las 
pendientes del plano $\left(\mathrm{C}_{\mathrm{B}}\right.$ y $\left.\mathrm{C}_{\mathrm{C}}\right)$ y la ordenada al origen $\left.\left(\mathrm{C}_{\mathrm{A}}\right): \hat{\beta}=\left(\mathrm{A}^{\mathrm{T}} \mathrm{A}\right)^{-1}\left(\mathrm{~A}^{\mathrm{T}} \mathrm{b}\right) \quad 5\right) ; \hat{\beta}=$ $\left[\begin{array}{l}\hat{\beta}_{1} \\ \hat{\beta}_{2} \\ \hat{\beta}_{3}\end{array}\right]=\left[\begin{array}{l}C_{A} \\ C_{B} \\ C_{C}\end{array}\right.$

\section{Cálculo de cuadrícula homogénea}

Si el levantamiento fue mediante cuadrícula, ésta ya fue trazada en campo al hacer el levantamiento topográfico, pero si fue por radiaciones, la cuadricula se crea a partir de los límites calculados previamente y con una equidistancia regular de la cuadrícula asignada para el cálculo de tamaño $\mathrm{L}_{\text {cuadricula }}(\mathrm{m})$, que puede ser la misma de la cuadrícula regular u otra según sea el caso. Con esto se definen el número de nodos en X y en Y (Figura 2).

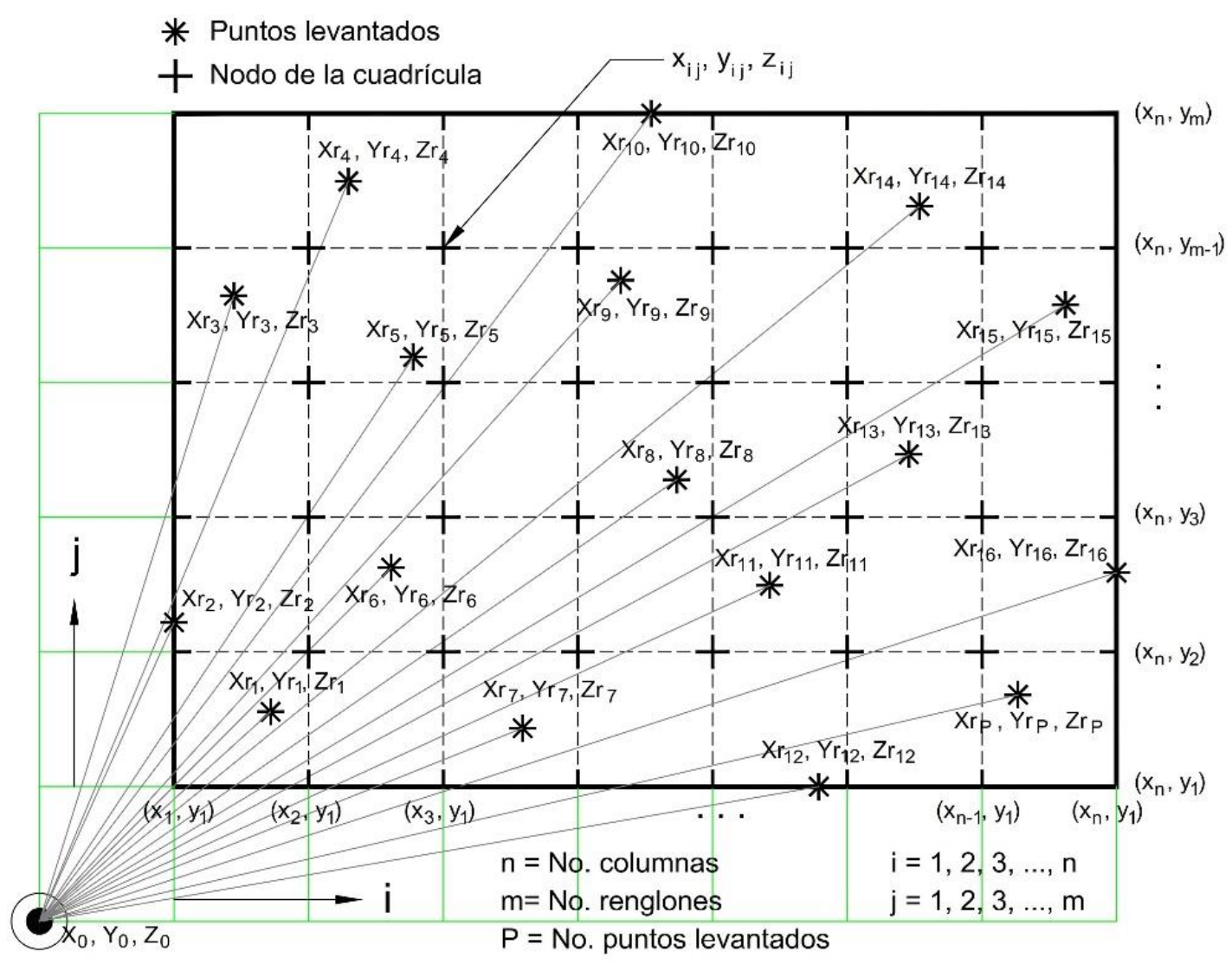

Figura 2. Elementos para determinar la cuadricula homogenea, a partir de un levantamiento topográfico por radiaciones y un MDE.

Los problemas que se detectaron en este punto se dan por tener terrenos muy cortos donde solo pueda definirse una linea, y por tanto, no se alcance a generar ningun cuadro, la solución más

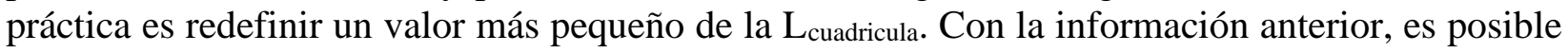
determinar la localización $\left(\mathrm{x}_{\mathrm{i}}, \mathrm{y}_{\mathrm{j}}\right)$ de cada nodo dentro de la cuadrícula. 


\section{Determinación de las coordenadas $\mathrm{z}_{\mathrm{ij}}$}

En cada nodo $(i, j)$ de la cuadrícula se determinan los valores de $z_{i j}$ a través un proceso geoestadístico de interpolación espacial, partiendo de la informacion levantada en campo $\left(\mathrm{Xr}_{\mathrm{k}}, \mathrm{Yr}_{\mathrm{k}}\right.$, $\mathrm{Zr}_{\mathrm{k}}$ ) de los datos de campo definidos como puntos vecinos al nodo (i, j). La definición de puntos vecinos se realiza calculando la distancia de cada nodo $(\mathrm{i}, \mathrm{j})$ a todas las coordenadas obtenidas en el levantamiento topográfico $\left(\mathrm{Xr}_{\mathrm{k}}, \mathrm{Yr}_{\mathrm{k}}, \mathrm{Zr}_{\mathrm{k}}\right)$, los $\mathrm{NC}$ puntos con las distancias más pequeñas conformarán el conjunto de puntos vecinos o cercanos para la estimación de $\mathrm{z}_{\mathrm{ij}}$, mediante la ecuación (7). $\mathrm{z}_{\mathrm{ij}}=\sum_{\mathrm{q}=1}^{\mathrm{NC}} \mathrm{W}_{\mathrm{q}} \mathrm{Z}_{\mathrm{q}}$ 7).

Donde: $\mathrm{z}_{\mathrm{ij}}=$ dato estimado en el nodo $(\mathrm{i}, \mathrm{j})$ de la cuadrícula generada de dimesiones $\mathrm{mxn} ; \mathrm{i}=1,2$, $3, \mathrm{n} ; \mathrm{j}=1,2,3, \ldots, \mathrm{m} ; \mathrm{n}=$ número de hileras; $\mathrm{m}=$ número de columnas. $\mathrm{Z}_{\mathrm{q}}=$ coordenada $\mathrm{Zr}_{\mathrm{k}}$ del punto de campo ubicado en la posición q del conjunto de puntos vecinos o cercanos. $\mathrm{W}_{\mathrm{q}}=$ peso o factor de ponderación calculado para el sitio q del conjunto de puntos vecinos o cercanos para la estimación de $z_{i j}$ en el nodo $(i, j)$. $N C=$ número total de puntos vecinos o cercanos al nodo $(i, j)$, que se utilizarán para estimar el dato $\mathrm{z}_{\mathrm{ij}}$. La suma de los valores de los pesos o factores de ponderación $\left(\mathrm{W}_{\mathrm{q}}\right)$ del conjuto de puntos vecinos o cercanos al nodo $(\mathrm{i}, \mathrm{j})$ deben cumplir la restricción definida en la ecuación (8): $\sum_{\mathrm{q}=1}^{\mathrm{NC}} \mathrm{W}_{\mathrm{q}}=1 \quad 8$ ).

\section{Cálculo de los pesos o factor de ponderación $\left(W_{q}\right)$}

El cálculo de lo pesos realiza aplicando el método de Kriging. Clayton y André (1998), indican que la escencia de Kriging es la estimación del semivariograma (Figura 3), en éste caso de elevaciones, el cual se construye definiendo 20 clases de distancias con todos los datos de campo (P) y calculando $\gamma(\mathrm{h})$ para cada clase ubicada a una distancia $\left(\mathrm{d}_{\mathrm{t}+\mathrm{h}}\right)$ el valor del semivariograma $\gamma(\mathrm{h})$ através de la ecuación 9. $\gamma(\mathrm{h})=\frac{\sum_{\mathrm{t}=1}^{\mathrm{P}}\left[\operatorname{Zr}\left(\mathrm{d}_{\mathrm{t}}\right)-\operatorname{Zr}\left(\mathrm{d}_{\mathrm{t}+\mathrm{h}}\right)\right]^{2}}{2 \mathrm{P}}$ 9)

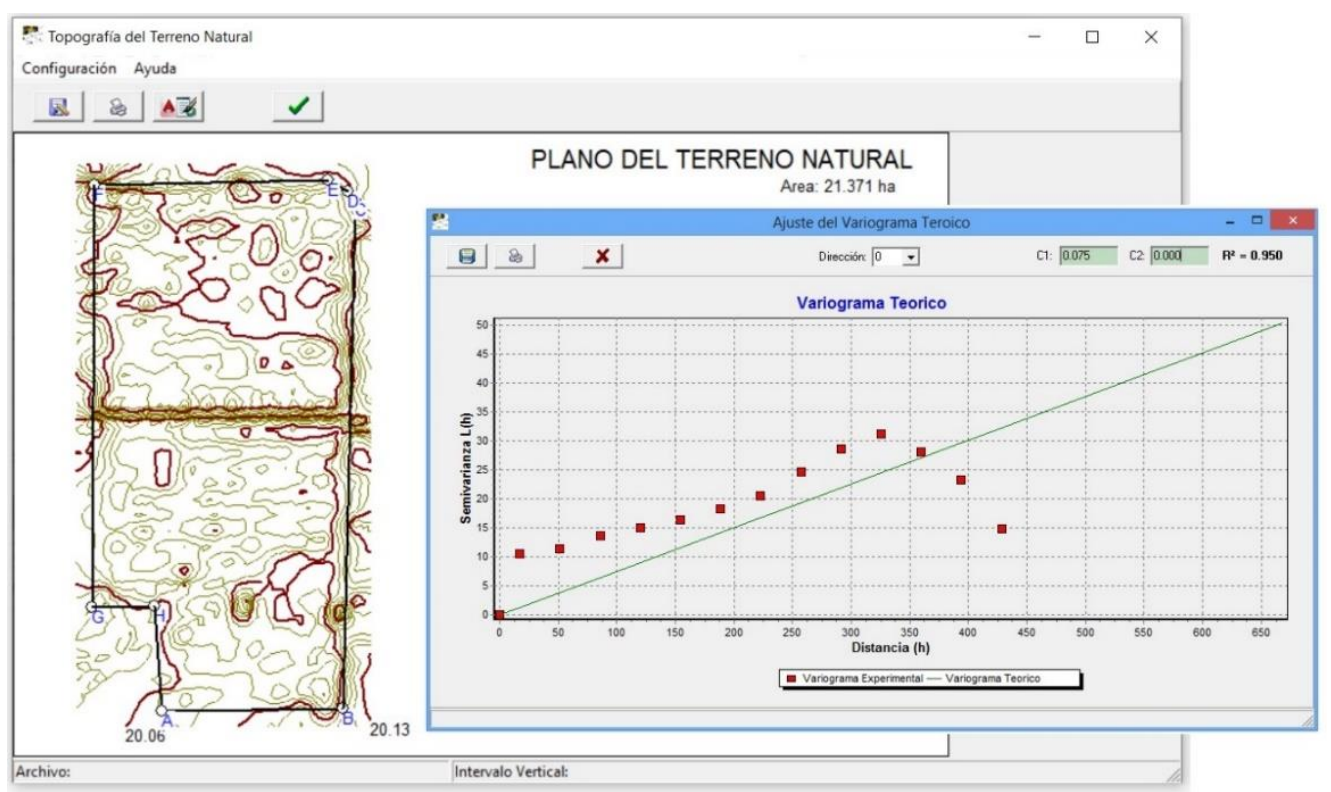

Figura 3. Topografía del terreno natural, aplicando el método de Kriging, en un levantamiento topográfico de distribución variable por radiaciones. 
Donde: $\mathrm{Zr}\left(\mathrm{d}_{\mathrm{t}}\right)=$ valor de la cota topografía $(\mathrm{Z})$ en el punto $\mathrm{t} ; \mathrm{Zr}\left(\mathrm{d}_{\mathrm{t}+\mathrm{h}}\right)=$ valor de la cota topográfica (Z) en el sitio ubicado a la distancia h del punto $t$; $P=$ número total de puntos del levantamiento por radiaciones.

Calculado el semivariograma para cada una de las distancias definidas en las clases, se ajusta un modelo teorico (Cisneros-lturbe et al., 1999), que pude ser: lineal, cuadrático, esférico, exponencial o gaussiano (Cuadro 1) ecuaciones 10, 11, 12, 13 y 14.

Cuadro 1. Modelos matemáticos (Delhome, 1978; Clark, 1979), disponibles en Terra para el ajuste del variograma teórico, a partir del semivariograma experimental.

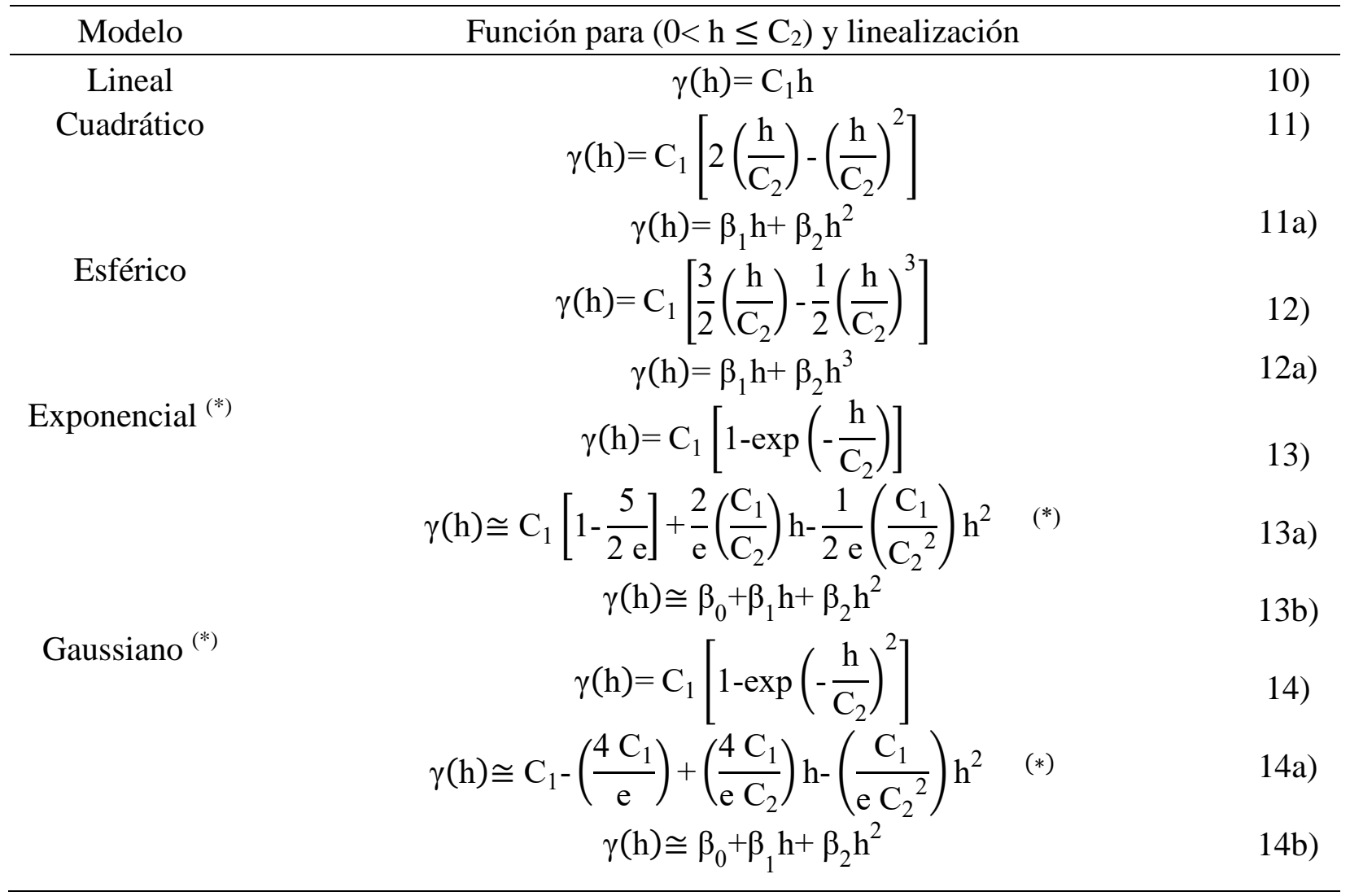

Cuando $\mathrm{h}>\mathrm{C}_{2}: \gamma(\mathrm{h})=\mathrm{C}_{1}$ (para los modelos cuadrático y esférico); ${ }^{(*)}=$ función aproximada a polinomio, mediante series de Taylor y para $\mathrm{h}=\mathrm{C}_{2}$.

Michael y Clayton (2014), mencionan que los comportamientos en el origen de los modelos pueden considerar o no el afecto pepita; es decir, la intersección con el eje Y o bien, considerar que salen del origen, como es el caso de la topografía. Inicialmente se probó con modelos de varigrama que incluyen el efecto pepita; sin embargo, la mayoria de soluciones implicaba valores negativos para el coeficiente $\mathrm{C}_{0}$, razon por la cual, se decidió utilizar el modelo que sale del origen (Legrá-Lobaina et al., 2010).

El cálculo de los coeficientes de los modelos $\left(\mathrm{C}_{0}, \mathrm{C}_{1}\right.$ y $\left.\mathrm{C}_{2}\right)$ se hizo con el metodo Newton-Raphson para la solución de sistemas de ecuaciones no lineales (Chapra y Canale, 2015), con resultados poco favorables, por lo que, los modelos se linealizaron (Cuadro 1) ecuaciones 11a, 12a, 13b y 14b, mejorando las soluciones. 
Antes de la linealización, los modelos exponencial y Gaussiano se aproximaron a una función polinomial (ecuaciones 13a y 14a), mediante series de Taylor (Purcell y Varberg, 2009). Una vez linealizados los modelos se aplicó la ecuación (5) para determinar los coeficientes $\beta_{\mathrm{i}}$ y para el calculo de los coeficientes $\mathrm{C}_{1}$ y $\mathrm{C}_{2}$ del variograma, las soluciones se listan en el (Cuadro 2) ecuaciones 15 a $20 \mathrm{~b}$.

\section{Cuadro 2. Coeficientes $\mathbf{C}_{1}$ y $\mathbf{C}_{2}$, obtenidos para determinar el variograma teórico.}

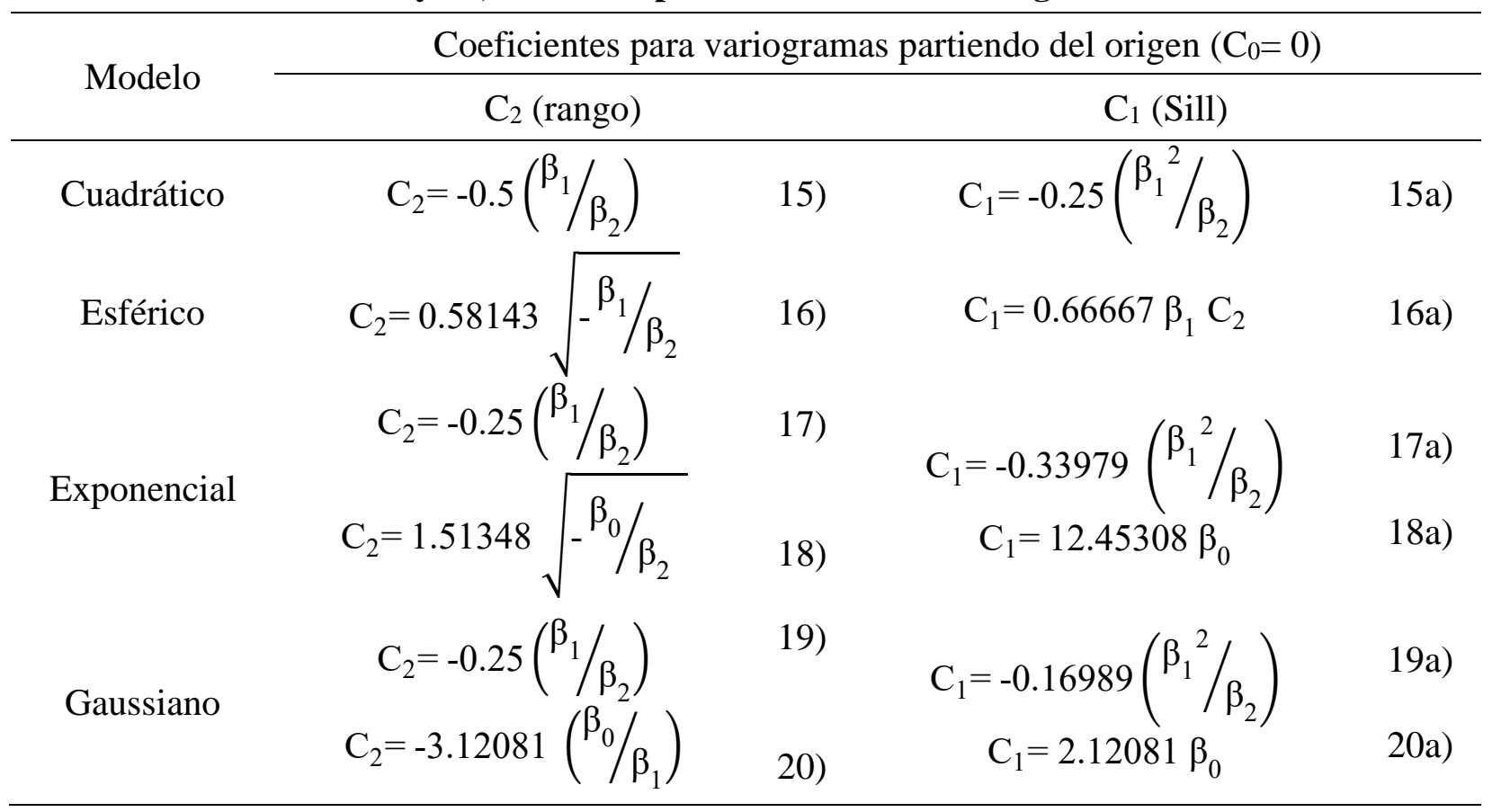

Una vez calculados los coeficientes del variograma teórico, la estimación de los pesos o factores de ponderación $\left(\mathrm{W}_{\mathrm{q}}\right)$ para cada uno de los nodos se hace mediante un proceso matricial (ecuaciones 21 y 22).

$$
\mathrm{Sw}=\mathrm{b} \text { 21) } \mathrm{S}=\left[\begin{array}{ccccc}
\gamma_{11} & \gamma_{12} & \cdots & \gamma_{1 \mathrm{n}} & 1 \\
\gamma_{21} & \gamma_{22} & \cdots & \gamma_{2 \mathrm{n}} & 1 \\
\vdots & \vdots & \ddots & \vdots & \vdots \\
\gamma_{\mathrm{n} 1} & \gamma_{\mathrm{n} 2} & \cdots & \gamma_{\mathrm{nn}} & 1 \\
1 & 1 & \cdots & 1 & 0
\end{array}\right] ; \quad \mathrm{W}=\left[\begin{array}{c}
\mathrm{W}_{1} \\
\mathrm{~W}_{2} \\
\vdots \\
\mathrm{W}_{\mathrm{n}} \\
\lambda
\end{array}\right] ; \quad \mathrm{b}=\left[\begin{array}{c}
\gamma_{1} \\
\gamma_{2} \\
\vdots \\
\gamma_{\mathrm{n}} \\
1
\end{array}\right]
$$

Donde: $\mathrm{S}=$ es una matriz cuadrada de orden $\mathrm{n} \times \mathrm{n}$; cada elemento de la matriz se calcula evaluando el variograma teorico con la distancia entre puntos; por ejemplo: el elemento $\gamma_{12}$ es calculado al evaluar la función del variograma con la distancia existente entre el punto 1 y 2 de los NC puntos cercanos o vecinos que se involucraron para el proceso de interpolación. W y b= son los vectores de orden $\mathrm{n} \times 1$.

El vector b se obtiene al evaluar la función del variograma con la distancia que hay entre el nodo (i, j) donde se estimará $\mathrm{z}_{\mathrm{ij}}$ y el dato vecino q, w contiene las n icógnitas involucradas en el cálculo; es decir, los pesos de cada uno de los NC datos, más un multiplicador de Lagrange $(\lambda)$ que 
almacena el error residual calculado en el proceso, así al despejar el vector w se obtendrán los pesos o ponderaciones buscadas $\left(\mathrm{W}_{\mathrm{q}}\right) \mathrm{w}=\mathrm{S}^{-1} \mathrm{~b}(23)$. Como producto de este paso se obtiene un archivo (*.MLL) con la información $\mathrm{z}_{\mathrm{ij}}$ de cada uno de los nodos de la cuadrícula homogénea.

\section{Ajuste de la ordenada al origen $\left(\mathbf{C}_{A}\right)$}

El ajuste de la ordenada al origen $\left(\mathrm{C}_{\mathrm{A}}\right)$ del plano proyecto calculado en el segundo paso, se realiza de acuerdo a la relación entre los cortes y rellenos definidos por el coeficiente de abundamiento $(\mathrm{R}=$ corte/relleno), la mayoría de autores coiciden que el coeficiente de abundamiento $(\mathrm{R})$ se debe encontrar en el rango: $1.1 \leq \mathrm{R} \leq 1.5$ (Walker 1988).

En la práctica, donde existieron rellenos habrá compactación y quedarán hoyos, estos se presentarán a través del tiempo por causa de la lluvia y compactación del suelo, por lo que es necesario, aumentar los cortes, por lo tanto disminuirán los rellenos esto se hace hasta tener un coeficiente de abundamiento (R) dentro del rango deseado, el ajuste se realiza bajando o subiendo el plano proyecto en $\pm \Delta \mathrm{h}$, h es el valor de incremento o decremento en la elevación del plano en $\mathrm{m}$, dependiendo del suelo y su mayor o menor contenido de materia orgánica.

Es decir, si un suelo es arenoso el coeficiente R será cerca de 1.1, arcilloso el valor del coeficiente será cerca de 1.5. Si se considerara un suelo intermedio (franco arcilloso) los valores del coeficiente variarán entre 1.20 y 1.30 (Walker, 1988). El cálculo de los vólumenes de corte y relleno se hace mediante el método de los 4 puntos; es necesario contar con una cuadrícula con los datos de campo o generada por el método de Kriging.

La incorporación del método de Kriging para la estimación de la cota $\left(\mathrm{z}_{\mathrm{ij}}\right)$ en cada nodo (i, j) de la cuadrícula es un procedimiento nuevo en nivelación de tierras y ha mostrado en la práctica resultados adecuados en el cálculo de volúmenes de corte y relleno. Como resultado de paso se obtiene un coeficiente $\mathrm{C}_{\mathrm{A}}$ ajustado $\left(\mathrm{C}_{\mathrm{Aajus}}\right)$, para la ecuación del plano proyecto. Con lo anterior, se calculan valores finales de espesores de cortes o rellenos para cada nodo $(i, j)$ de la cuadrícula y por tanto volúmenes de cortes y rellenos, así como costos de nivelación por hectárea y totales.

\section{Generación y dibujo de isolíneas}

Se elaboran planos de curvas de nivel para el terreno natural (configuración antes de la nivelación) y para el proyecto de terreno nivelado (configuración después de la nivelación). Éste proceso se realiza de dos formas: a) se crean curvas de nivel para todo el terreno, sin importar como fue realizado el levantamiento topográfico se crea una nueva cuadrícula más fina ( $\left.{ }^{\prime}{ }_{i}, y^{\prime}{ }_{j}, z^{\prime}{ }_{i j}\right)$ generada con longitudes entre nodos más pequeña, de aproximadamente un cuarto la longitud de cuadrícula para la nivelación.

Ésta nueva cuadrícula se calcula, a partir de los datos de campo levantados en cuadrícula rectangular $\left(\mathrm{x}_{\mathrm{i}}, \mathrm{y}_{\mathrm{j}}, \mathrm{z}_{\mathrm{ij}}\right)$ o por radiaciones $\left(\mathrm{Xr}_{\mathrm{k}}, \mathrm{Yr}_{\mathrm{k}}, \mathrm{Zr}_{\mathrm{k}}\right)$ aplicando el proceso de interpolación espacial con el método de Kriging explicado en los puntos anteriores o el método del inverso de la distancia. Los límites de la nueva cuadrícula se extienden para cubrir perfectamente la poligonal del terreno y evitar áreas sin curvas. El cálculo y dibujo de isolíneas o curvas de nivel del terreno nivelado, se realiza con los nodos $(i, j)$ de la nueva cuadrícula, cuya cota $\mathrm{z}_{\mathrm{ij}}$ se obtiene con la 
ecuación final, ecuación (1) definida para el plano proyecto; y b) en caso de que por cambios abruptos de pendientes o tamaño del lote sea necesario dividir el terreno, para hacer su nivelación independiente por tablas, se diseñó un algoritmo que permite dividir el predio en dos partes de forma interactiva con el usuario.

En el ejemplo se muestra la topografía del terreno natural para que sobre ella se trace una línea divisoria que permita separar los puntos levantados en los nuevos polígonos y planos naturales de proyecto, que resultan con la línea divisoria. En caso de necesitar dividir el predio en más de dos partes se tiene una forma de captura para definir los polígonos.

Esto último con el fin de que el proyectista identifique manualmente los linderos de los polígonos y con esto queden dentro todos los puntos levantados en campo. Una vez definidas las tablas se aplicarán los seis puntos descritos anteriores para realizar el cálculo del plano natural de proyecto y final, así como el cálculo de volúmenes de corte y relleno en forma independiente de cada una de las tablas.

\section{Resultados y discusión}

Terra 1.0 se desarrolló con el lenguaje de programación Delphi (Pascal), diseñado para funcionar como herramienta en cualquier proyecto de nivelación de tierras donde la topografía sea levantada en cuadricula con trazo rectangular o radiaciones. El sistema permite la nivelación general de todo el predio levantado o la división por tablas.

El cálculo de volúmenes de corte y relleno se realiza con la cuadrícula rectangular aplicando el método de Kriging para el cálculo. La estimación de los valores de la cuadrícula genera valores congruentes con la topografía y por ende el cálculo de volúmenes se obtiene de acuerdo con los procedimientos técnicos preestablecidos.

\section{Captura de la información}

Terra, cuenta con tres cuadros de diálogo para la capturar la información de entrada: 'datos del proyecto y parámetros del diseño' para los datos generales, 'datos de la poligonal del terreno y cálculo del centroide' para capturar las coordenadas $\left(\mathrm{X}_{\mathrm{v}}, \mathrm{Y}_{\mathrm{v}}\right)$ de los vértices de la poligonal del terreno y 'datos topográficos para la nivelación', para los datos de campo con coordenadas $\left(\mathrm{x}_{\mathrm{i}}, \mathrm{y}_{\mathrm{j}}\right.$, $\left.\mathrm{Z}_{\mathrm{ij}}\right)$ o $\left(\mathrm{Xr}_{\mathrm{k}}, \mathrm{Yr}_{\mathrm{k}}, \mathrm{Zr}_{\mathrm{k}}\right)$ según el levantamiento topográfico realizado y la longitud de equidistancia de la cuadrícula.

\section{Topografía del terreno natural}

El programa permite generar y visualizar la topografía del terreno con curvas de nivel (Figura 6). Para poder dibujarlas se ha calculado un modelo digital de elevaciones usando el método de Kriging. Según Delhome (1979) recomienda el uso del método de Kriging en las aplicaciones a la hidrociencia, indicando que describe en forma adecuada el comportamiento de la topografía del terreno natural. La propuesta inicial para el cálculo de los coeficientes $\mathrm{C} 0$ y $\mathrm{C} 1$ del modelo del variograma teórico, se ajusta automáticamente al modelo lineal; sin embargo, se puede elegir otro en forma personalizada (Figura 3). 


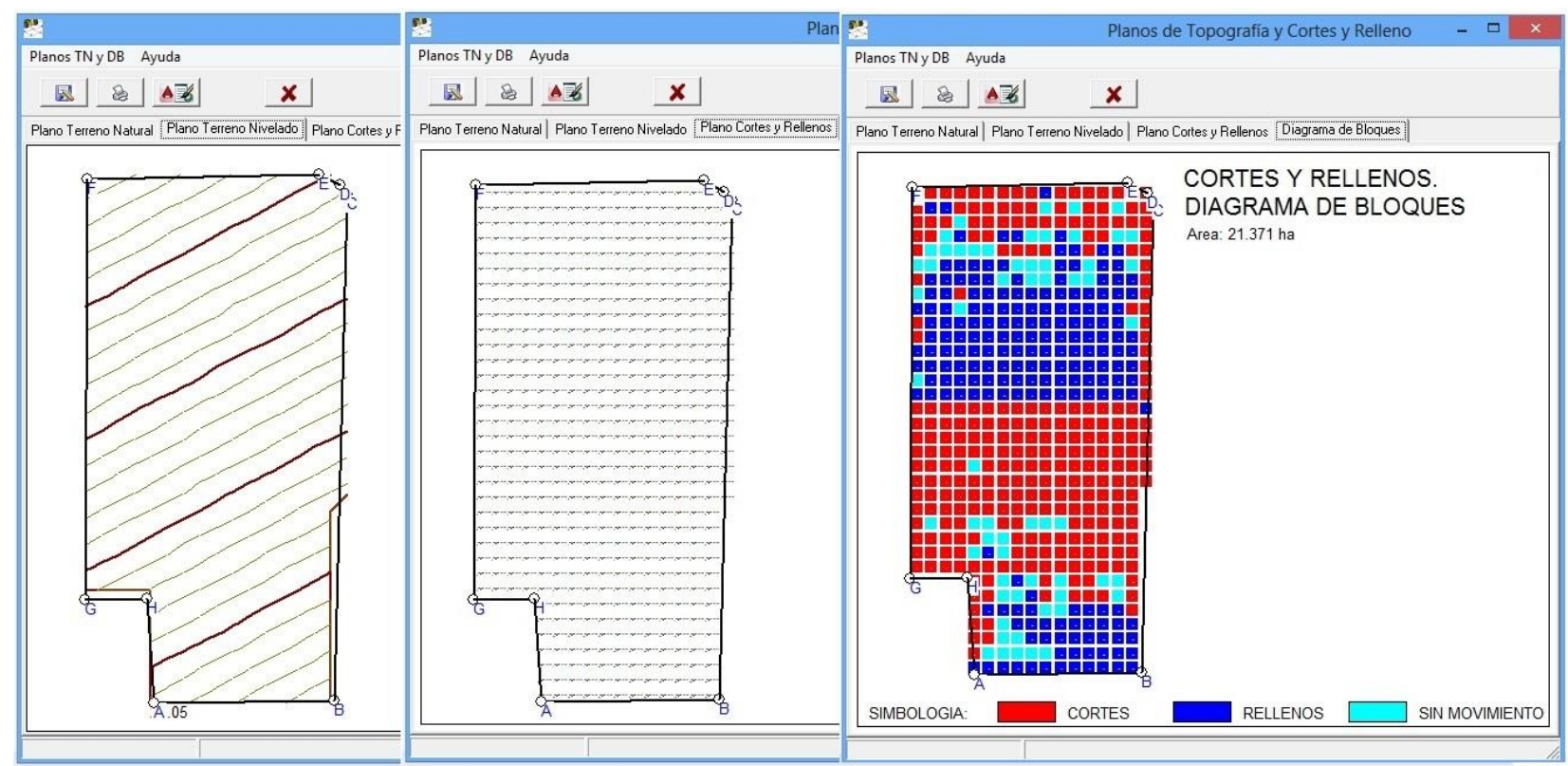

Figura 6. Planos de curvas de nivel del terreno nivelado, de valores de corte y relleno en nodos de la cuadrícula y diagrama de bloques de movimiento de tierras.

\section{Levantamiento topográfico en cuadrícula rectangular}

El software desarrollado permite procesar la información obtenida con un levantamiento con una cuadrícula rectangular, por lo que se puede aplicar la metodología tradicional para el cálculo de nivelación de tierras. En este caso ya existe la cuadrícula desde campo por lo que no hay necesidad de crear una nueva. Como es el caso del levantamiento por radiaciones donde se genera la cuadrícula.

\section{División del terreno en tablas}

El software desarrollado permite dividir el terreno en dos tablas, trazando una línea divisoria y generando los dos polígonos independientes en forma automática (Figura 4). Cuando se realiza un levantamiento por radiaciones, algunos puntos son tomados cerca de los linderos y con una alta probabilidad pueden caer fuera del terreno, el sistema asigna todos los puntos en alguno de los dos polígonos; sin embargo, cuando la división implica un mayor número de partes, será muy complicado agrupar los puntos, razón por la cual, se cuenta con una hoja de entrada de información donde el usuario debe ingresar las coordenadas de cada polígono y para cada tabla solo se extraerá la información que se encuentre dentro del polígono definido.

Una vez realizada la división del terreno, se analiza por separado cada tabla definida. En el ejemplo se realizó la nivelación por tablas, haciendo una divisoria en la parte media del terreno donde se puede ver claramente que hay un cambio de pendiente brusco, buscando reducir los volúmenes de corte y relleno y por ende el costo total de la nivelación (Figuras 4 y 5). 

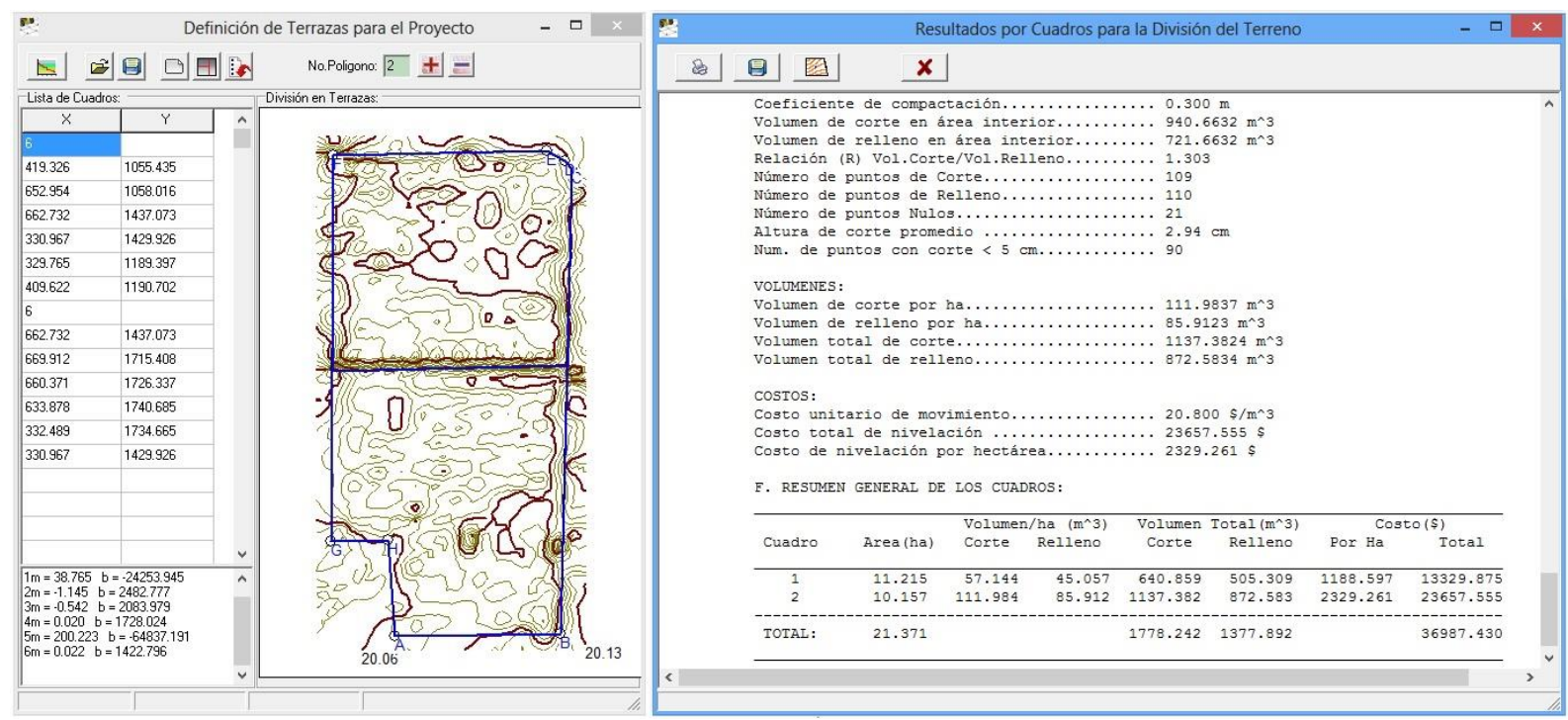

Figura 4. División del terreno en tablas independientes.
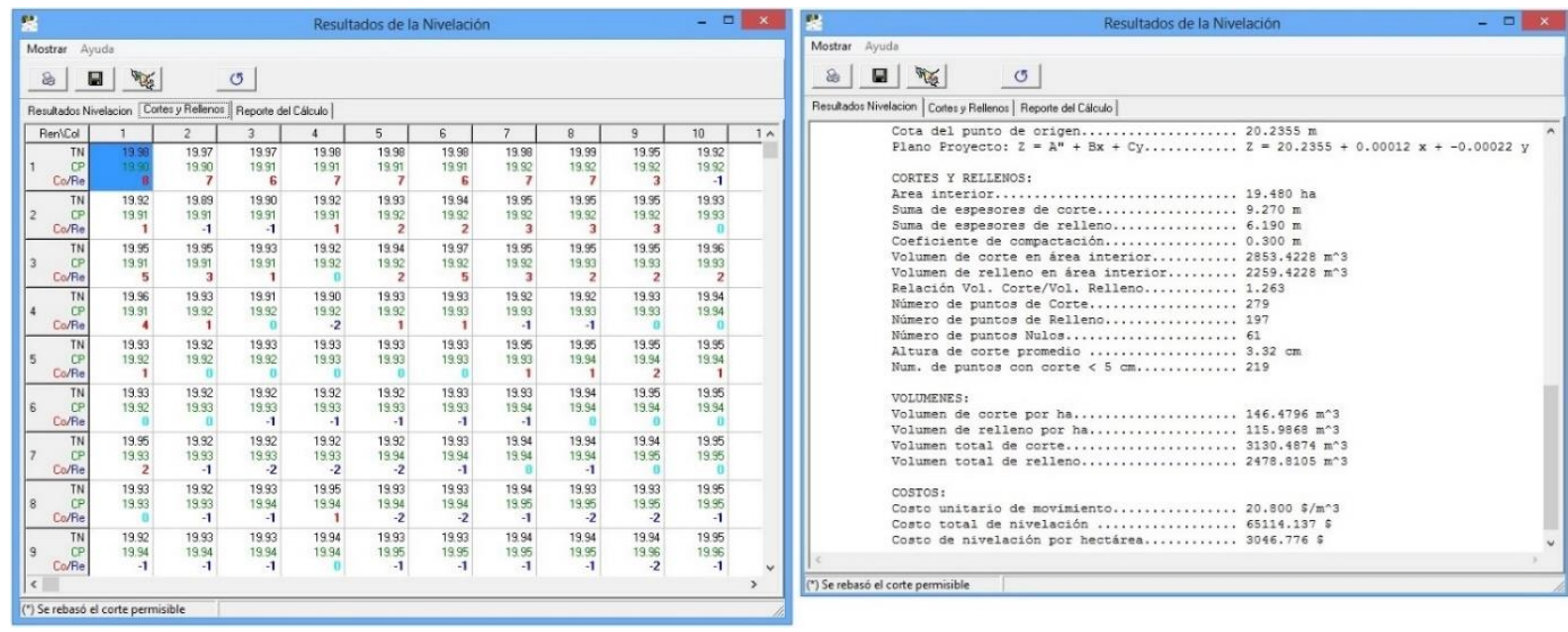

Figura 5. Resultados de la nivelación para el predio del ejemplo, sin divisiones.

\section{Resultados del cálculo del proyecto de nivelación}

Después de realizar los cálculos respectivos con los datos de entrada, Terra proporciona con características detalladas información de todo el proyecto, agrupado en tres cejas: resultados nivelación, cortes y rellenos y reporte o memoria de cálculo. Las secciones que se describen en los resultados son: datos generales del proyecto, parámetros del diseño, coordenadas de la poligonal y resultados de la nivelación (generales, ajuste al plano, cortes y rellenos, volúmenes y costos).

En la sección de cortes y rellenos, se presenta una tabla que en cada nodo (i,j) contiene, las coordenadas del terreno natural, cota del plano proyecto y el corte o relleno en centímetros de cada punto de la cuadrícula. Finalmente, se presenta la sección del reporte de la memoria con el cálculo 
desglosado de los resultados: la solución matricial del sistema de ecuaciones para calcular el plano proyecto, el cálculo del área, centroide y cota centroidal, el proyecto inicial con la información topográfica y del plano proyecto, el cálculo de la ecuación del plano y las iteraciones para el ajuste del plano (Figura 5).

\section{Cálculo y generación de isolíneas}

Con Terra se pueden calcular y dibujar las curvas de nivel representativas de la cuadrícula analizada. Para ello se crean las isolíneas de dos formas distintas: la primera con los datos de campo obtenidos en el levantamiento topográfico por cuadrícula, la segunda mediante el procedimiento geoestadístico de interpolación espacial detallado en las secciones anteriores, con el cual se crea una cuadrícula con uno de dos métodos: el método de Kriging o el del inverso de la distancia. También se presenta un plano de cortes y rellenos y un diagrama de bloques de cortes y rellenos que sirve como guía al tractorista para el trabajo de la nivelación en campo (Figura 6).

\section{Estimación de costos}

El costo total de nivelación para el ejemplo de las figuras 4 y 5, cuyo resumen se muestra en el Cuadro 3, tuvo una reducción de 43\%, de \$65 114.14 a \$ 36987.43 considerando el costo $/ \mathrm{m}^{3}$ de movimiento de tierra en $\$ 20.80$, al dividir el terreno en dos tablas basado en la topografía del terreno natural, lo cual mejoró el diseño inicial sin divisiones, considerando solamente pendientes naturales promedio.

Cuadro 3. Comparación de volúmenes y costos para el ejemplo, aplicando la nivelación al terreno completo vs el predio dividido en dos tablas según topografía del terreno natural.

\begin{tabular}{|c|c|c|c|c|c|c|c|}
\hline \multirow{2}{*}{$\begin{array}{l}\text { No. } \\
\text { Tablas }\end{array}$} & \multirow{2}{*}{ Área (ha) } & \multicolumn{2}{|c|}{ Volumen $\left(\mathrm{m}^{3}\right)$} & \multicolumn{2}{|c|}{ Volumen total $\left(\mathrm{m}^{3}\right)$} & \multicolumn{2}{|c|}{ Costo $(\$)$} \\
\hline & & Corte & Relleno & Corte & Relleno & $\begin{array}{c}\text { Por } \\
\text { hectárea }\end{array}$ & Total \\
\hline 1 & 21.371 & 146.48 & 115.987 & 3130.4874 & 2478.8105 & 3046.776 & 65114.137 \\
\hline \multirow[t]{3}{*}{2} & 21.371 & & & 1778.242 & 1377.892 & & 36987.43 \\
\hline & 11.215 & 57.144 & 45.057 & 640.859 & 505.309 & 1188.597 & 13329.875 \\
\hline & 10.157 & 111.984 & 85.912 & 1137.382 & 872.583 & 2329.261 & 23657.555 \\
\hline
\end{tabular}

El valor de 1 en núm. tablas, indica que el terreno no fue dividido.

Uno de los aspectos técnicos más importantes del presente trabajo es la generación del modelo digital de elevaciones mediante el método de Kriging, para la determinación de alturas de corte o relleno y cuantificación de los volúmenes de corte y relleno, donde se aplica el método de los cuatro puntos sugerido por el Departamento de Agricultura de Estados Unidos (1970) y que aplica para su estimación una cuadrícula cuya área de influencia sea un cuadrado. Para garantizar que el modelo de Terra, es técnicamente adecuado se comparó el DEM con un programa profesional como Surfer (Golden software, 2011), los modelos generados se muestran en la Figura 7. 


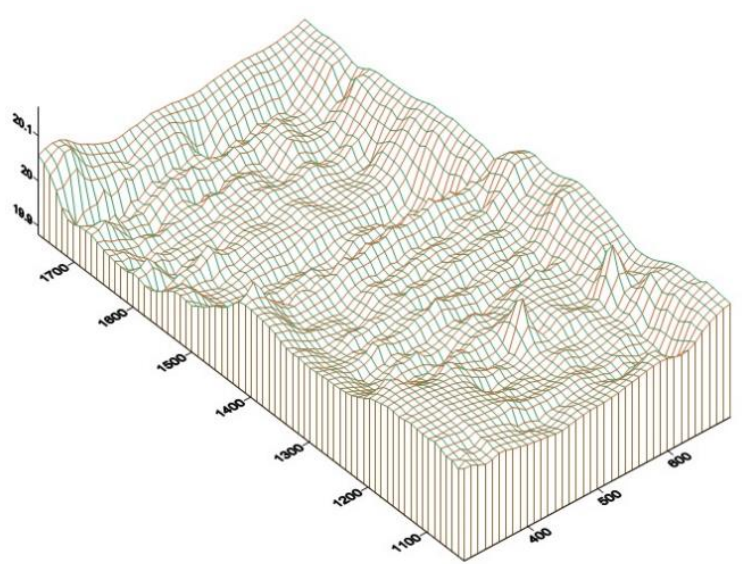

Modelo 3D, con los datos de SURFER

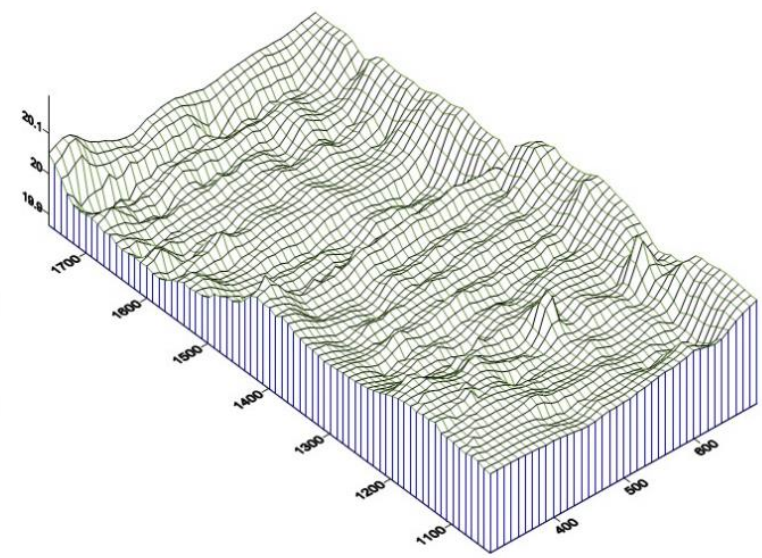

Modelo 3D con los datos de TERRA

Figura 7. Modelos digitales de elevación generados por Surfer y por Terra, para el ejemplo.

Los modelos constan de 39 columnas y 73 hileras, con un total de 2847 datos de elevaciones Z, con medias: Zm_ Terra $=20.00739094, Z_{-} m_{-}$SURFER $=20.00997930$ y varianzas: S $^{2}$ - Terra= 0.00373831, S²_SURFER: 0.00388370, al practicarles una prueba estadística para medias diferentes y varianzas distintas se concluyó que los modelos anteriores con $97 \%$ de confiabilidad tienen valores de medias iguales y con un valor de $99 \%$ de confiabilidad tienen varianzas similares, lo que nos hace concluir que son prácticamente iguales.

Lo anterior, permite aseverar que el modelo generado por Terra, predice el comportamiento en forma adecuada y por tanto al generar la cuadrícula para estimar los valores de cortes y rellenos genera volúmenes congruentes que permiten ajustar el plano proyecto de la nivelación de forma adecuada. Con la aplicación de las tecnologías modernas para la nivelación de tierras, como es la nivelación con rayo láser, pareciera ser innecesario el desarrollo de un proyecto de nivelación.

sin embargo, no es así; cualquier proyecto de nivelación, independientemente de la forma en que se tomen los datos de campo, necesita un proyecto ejecutivo con datos y resultados que incluyan una memoria de cálculo con la estimación de volúmenes de corte y relleno y por supuesto el costo de la nivelación, el plano con la topografía original y el terreno nivelado. Esto será el sustento del proyecto y el respaldo para que el usuario realice los trámites necesarios en caso de requerir apoyos económicos en instituciones que lo brindan al campo o bien en el pago del movimiento de tierras al tractorista que realice el trabajo, sin duda alguna.

\section{Conclusiones}

Terra 1.0, que se presenta, es un sistema de cómputo electrónico desarrollado como herramienta fundamental para auxiliar a los técnicos en la formulación y optimización de proyectos ejecutivos de nivelación de tierras. Incluye algoritmos para el cálculo del proyecto a partir de un levantamiento topográfico realizado en cuadrícula o con distribución variable.

El uso del método de Kriging se integró con éxito para el cálculo de las isolíneas del plano del terreno natural, del terreno nivelado, y la utilización de una cuadrícula levantada o generada que permite realizar el cálculo del volumen de cortes y rellenos. La metodología utilizada, implicó el 
resolver en forma detallada el ajuste de mínimos cuadrados ordinarios usando un método matricial para el cálculo del plano inicial e implementar un algoritmo eficiente para el ajuste del coeficiente $\mathrm{C}_{\mathrm{A}}$ de la ecuación del plano.

En el caso de la cuadrícula generada, se incluyen algoritmos para la determinación de semivariogramas y se propusieron ecuaciones algebraicas para el cálculo de los coeficientes $\mathrm{C}_{1} \mathrm{y}$ $\mathrm{C}_{2}$ del variograma teórico, para el cálculo de los pesos en la interpolación espacial de los datos de la cuadrícula. En caso de la división en tablas o terrazas, se implementaron algoritmos para la separación de los puntos de campo según los polígonos divididos; se incluyen las rutinas necesarias para que el proyectista pueda generar un diseño técnicamente adecuado de la nivelación, con lo que se logra la disminución de los volúmenes totales de cortes y rellenos y por tanto se logra disminuir los costos de nivelación. El sistema Terra, se podrá utilizar con las nubes de puntos que se generan en levantamientos topográficos que se realizan con VANTs, conocidos como drones.

\section{Literatura citada}

Cano-Muñoz, J. y Vázquez-Guzmán, A. 1997. Nivelación de tierras. Ediciones Mundi-Prensa. Ministerio de Agricultura, Pesca y Alimentación, Sevilla, España. 215 p.

Cisneros-lturbe, H. L.; Bouvier, C. y Domínguez-Mora, R. 1999. Aplicación del método Kriging en la construcción de campos de tormenta en la ciudad de México. Ingeniería Hidráulica en México. 16(3):5-14.

Clark, I. 1979. Practical geostatistics. Cuarta reimpresión. (Ed.). Elsevier Applied Science. London, Great Britain. 127 p.

Clayton, V. D. and André, G. J. 1998. GSLIB: geoestatistical software library and User's Guide. Oxford University Press, New York, USA. 369 p.

CONAGUA. 2014. Subdirección General de Infraestructura Hidroagrícola. Gerencia de Distritos de Riego. Proyecto de riego por gravedad tecnificado (RIGRAT). Curso de capacitación: Nivelación de tierras para el riego por gravedad. México, DF. 132 p.

Covarrubias-Lugo, A. 1968. Cálculo de nivelación de tierras para riego por medio del cómputo electrónico. Ingeniería Hidráulica. México, DF. 80 p.

Chapra, C. S. y Canale, R. P. 2015. Métodos Numéricos para Ingenieros. Editorial Mc Graw Hill. Séptima Edición. México, DF. 500 p.

Chávez-Morales, J.; Ibañez-Castillo, L. A. y Hernández-Saucedo, F. R. 1990. Programa de nivelación de tierras (NIVTERRA 1.1). Colegio de Postgraduados, Hidrociencias. Montecillo, México. 25 p.

Delhome, J. P. 1978. Kriging in the hydrociencies. Advances in water resouces.1(5):251-266.

Fuentes-Ruiz, C. y Rendón-Pimentel, L. 2012. Riego por gravedad. Universidad Autónoma de Querétaro-CONAGUA. 358 p.

Guajarati, D. N. y Porter, D. C. 2010. Econometría. (Ed.). Mc Graw Hill. Quinta Edición. México, DF. 801-876 p.

Hernández-Saucedo, F. R y Sánchez-Bravo, J. R. 2008. Manual para diseño de obras de riego pequeñas. Capítulo 1.4: Nivelación de Tierras. Editado por: De León, M. B. y Robles, R. B. D. Instituto Mexicano de Tecnología del Agua (IMTA). Jiutepec, Morelos. 53-73 p.

Legrá-Lobaina, A. A. y Atanes-Beatón, D. M. 2010. Variogramas adaptativos: un método práctico para aumentar la utilidad del error de estimación por Kriging. Minería y Geología. 26(4):53-78. 
Marr, J. C. 1957. Grading land for surface irrigation. University of California, Division of Agricultural Sciences. Davis, USA. Circular 438. 55 p.

Michael, J. P. y Clayton, V. D. 2014. Geostatistical reservoir modeling. Second Edition. Oxford University Press. New York, USA. 362 p.

Montgomery, D. C. y Runger, G. C. 2008. Probabilidad y estadística aplicadas a la ingeniería. Editorial Limusa Wiley. $2^{\text {da. }}$ (Ed.). México, DF. 430-560 pp.

Purcell, E. J y Varberg, D. 2009. Cálculo con geometría analítica. Editorial Prentice Hall Hispanoamericana, SA. $9^{a}$ Edición. México, DF. 451 p.

Saucedo, H.; Fuentes, C. y Zavala, M. 2006. El sistema de ecuaciones de Saint-Venant y Richards del riego por gravedad: 3 . Verificación numérica de la hipótesis del tiempo de contacto en el riego por melgas. Ingeniería Hidráulica en México. 21(4):135-143.

US. 1970. Department of Agriculture, Soil Conservation Service. Land leveling, national engineering handbook. US. Government Printing Office. Washington, DC. Chap 12:65 p.

Villatoro, M.; Henríquez, C. y Sancho, F. 2008. Comparación de los interpoladores IDW y Kiging en la variación espacial de PH, CA, CICE y P del suelo. Agron. Costarricense. 32(1):95-105.

Walker, W. R. and Skogerboe, G. V. 1987. Surface irrigation: theory and practice. Editorial Prentice-Hall. A Division of Simon and Schuster. Englewood Cliffs, New Jersey, 07632 USA. 178-191 pp. 